\title{
The frequency of dental caries in adult patients with gastroesophageal reflux disease
}

\author{
Sîmpălean DS*, Ghiga Dana, Petrișor M, Mărușteri M, Bacârea V, Bățagă Simona \\ University of Medicine and Pharmacy of Tirgu Mureș, România
}

\begin{abstract}
Introduction. The aim of the study was to evaluate the frequency of dental caries in adults patients with gastroesophageal reflux disease. Material and method. A cross-sectional study was performed between November 2013 and October 2014. All subjects who agreed to participate to the study were asked to complete a questionnaire regarding personal information, oral hygiene and dental evaluation. The individuals with complete false teeth (superior and inferior removable prosthetic devices) were excluded from the study. The study included 134 people divided in two groups, based on upper gastrointestinal endoscopy: a group with gastroesophageal reflux disease (71), and a control group (63). Dental evaluation was performed by a dentist blind to the diagnosis of the subjects. Dental caries were evaluated by applying the decayed, missing, and filled teeth index (DMFT index). The statistical significance was defined as $p<0.05$. Results. From 71 subjects included in GERD group, 33 (46.48\%) were males and 38 females (53.52\%) with a mean age of $44.99 \pm 11.19(42,59.15 \%$ from urban area and 29, $40.85 \%$, from rural area). In the control group we included 28 (44.44\%) males and 35 (55.56\%) females (mean age 43.84 \pm 9.48$)$ and 29 (46.03\%) subjects were from urban area and 34 (53.97\%) from rural area. DMFT index in GERD group was $19.49 \pm 4.28$ and in control group $18.16 \pm 4.54(p<0.05)$. Conclusions. The present study showed that there is no difference between GERD and control group, regarding the frequency of dental caries.
\end{abstract}

Key words: oral manifestations, dental caries, gastroesophageal reflux

Received: 10 December 2014 / Accepted: 3 May 2015

\section{Introduction}

Gastroesophageal reflux is a physiological process, which consists in an effortless retrograde movement of the gastric contents to the esophagus (1). The gastroesophageal reflux disease (GERD) is a common chronic disease $(2,3)$, with a high prevalence (4) and an increasing incidence (5). GERD occurs when the amount of gastric reflux into the esophagus is above the normal range, causing various symptoms, which can involve esophageal mucosal injury (esophagitis) $(5,6)$. Regurgitation, pyrosis (heartburn), which can be reported in the presence or absence of regurgitation, retrosternal pain, dysphagia, sour taste and odynophagia, are considered typical manifestation of $\operatorname{GERD}(7,8)$. Oral manifestations that may occur in patients with gastroesophageal reflux disease include periodic hypersalivation, xerostomia (dry mouth syndrome), burning sensation, halitosis, and dental erosions. A certain pattern of erosion was observed in patients affected by this disease: they appeared more frequently on the occlusal surfaces of posterior mandibular teeth and lingual surfaces of the anterior maxillary teeth (10).

Dental caries are represented by tooth hard tissue demineralization and destruction, caused by bacteria and acid. During a day, the enamel demineralizes and remineralizes many times. When this balance is disrupted and demineralization exceeds remineralization, the caries progress. In our country, dental caries occur frequently from an early

* Correspondence to: Dan Ștefan Sîmpălean

E-mail: dansampalean@gmail.com age. The pathogenesis implies both internal and external factors.

Dental caries can have serious and long term complications, which is why they have to be treated properly, but most important they should be prevented.

The aim of the study was to evaluate the frequency of dental caries, in adult patients with gastroesophageal reflux disease.

\section{Material and method}

A cross-sectional study was performed, conducted between November 2013 and October 2014. During the study period all individuals with ages between 20 and 80 years old, who were hospitalized to the Gastroenterology clinic of the Emergency County Clinical Hospital, were evaluated. All subjects who agreed to participate in the study were asked to fill in a questionnaire regarding personal information, oral hygiene and dental evaluation.

Besides personal information, the questionnaire contained 7 questions, 5 questions concerning oral hygiene (fig. 1) and 2 questions referring to dental evaluation (Fig. 2). Regarding oral hygiene, we obtained information on the frequency of teeth brushing, the oral care products used and how often the respondent visits a dentist.

Concerning dental evaluation, we were interested if the respondent complained regarding the dental status and dental treatments.

After filling in the questionnaire, there were excluded from the study the individuals with complete false teeth (superior and inferior removable prosthetic devices). We 
Fig. 1. Questionnaire regarding oral hygiene

\begin{tabular}{l} 
1. Please indicate how many times you brush your teeth: \\
$\square 1$ time / day \\
$\square 2$ times / day \\
$\square$ Few days a week \\
$\square$ I do not brush my teeth \\
2. What type of toothbrush bristles do you use? \\
$\square$ Extra soft \\
$\square$ Soft \\
$\square$ Medium \\
$\square$ Hard \\
3. What oral care products do you use? \\
$\square$ Toothpaste \\
$\square$ Toothpasteand mouthwash \\
$\square$ Toothpasteand dental floss \\
$\square$ Toothpaste andmouthwash and dental floss \\
$\square$ I do not use oral care products \\
4 . How often do you use other oral care products(mouthwash or dental \\
floss)? \\
$\square 1$ time / day \\
$\square$ times / day \\
$\square$ Few days a week \\
$\square$ Never \\
5 . How often do you visit a dentist during a year? \\
$\square 1$ time / year \\
$\square 2$ times / year \\
$\square$ Few times \\
$\square$ Only when I have a toothache \\
\hline
\end{tabular}

Fig. 2 - Questionnaire regarding dental evaluation.

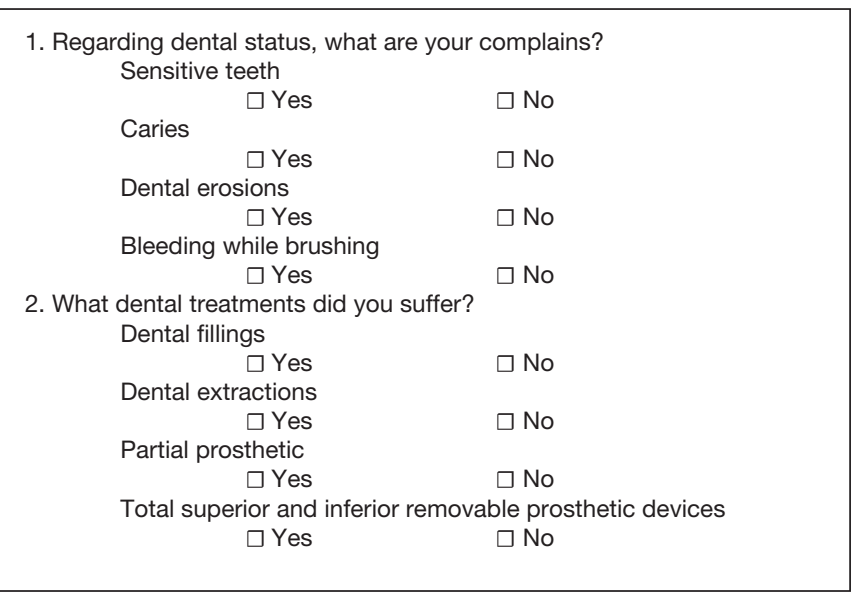

included in the study 134 people divided in two groups, based on upper gastrointestinal endoscopy: a group with gastroesophageal reflux disease $(n=71)$, and a control group $(\mathrm{n}=63)$.

Dental evaluation was performed by a dentist blind to the diagnosis of the subjects. Dental caries were evaluated by applying the decayed, missing, and filled teeth index (DMFT index). The diagnosis of caries was established by visual inspection and tactile examination.

Visual inspection was done on a clean tooth, previously dried with air flow and using the proper lighting. Tactile examination was made with a blunt dental probe. The blunt dental probe was used to remove plaque and food debris and to determine whether a pit or a fissure "clings". The diagnosis of dental caries is established if there are discolored areas (small white lesions on smooth surfaces) or light to dark brown lesion in pits or fissures and visible cavities and if the dental probe clings.

For data analysis there were used elements of descriptive and inferential statistics. Central tendency comparison was performed applying unpaired T test with Welch's correction test and Mann Whitney test for unpaired data. We chose the significance level of 0.05 . Statistical analysis was performed using GraphPad Prism 5 trial version.

\section{Results}

From 71 subjects included in the GERD group, 33 (46.48\%) were males and 38 females $(53.52 \%)$, and in the control group were included $28(44.44 \%)$ males and 35 (55.56\%) females (Figure 3).

Regarding environment, in the GERD group were included 42 individuals, $59.15 \%$, from urban area and $29,40.85 \%$, from rural area. In the control group, 29 (46.03\%) subjects were from urban area and 34 (53.97\%) from rural area (Figure 4).

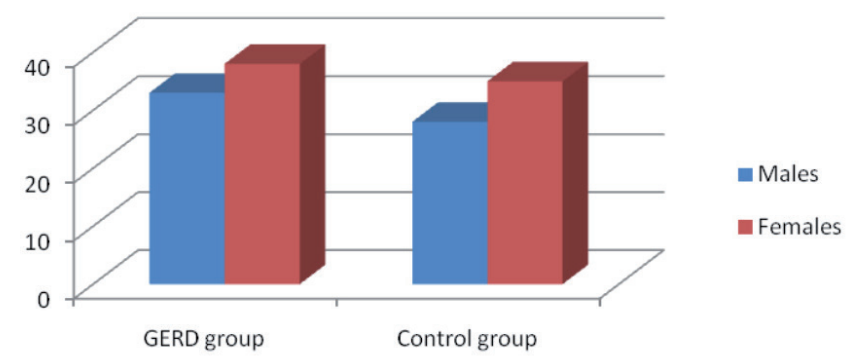

Fig. 3. Sex distribution in GERD and control group

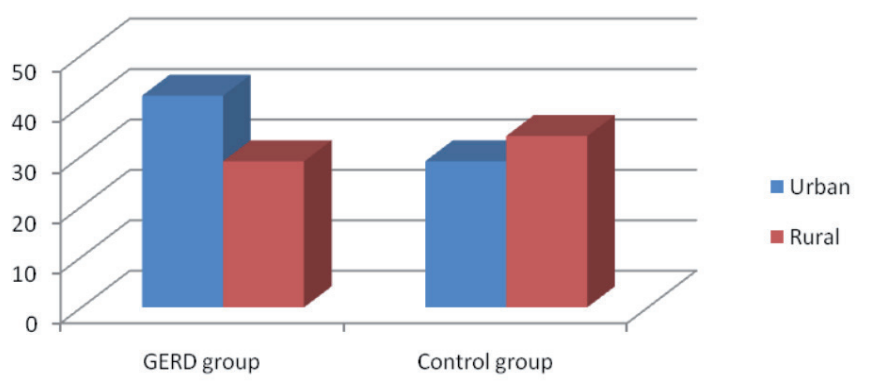

Fig. 4. Environment distribution in GERD and control group

Average age in GERD group was $44.99 \pm 11.19$ and in the control group the average age was $43.84 \pm 9.48$, with a value $\mathrm{p}=0.5229(\mathrm{p}>0.05)$ (Figure 5$)$.

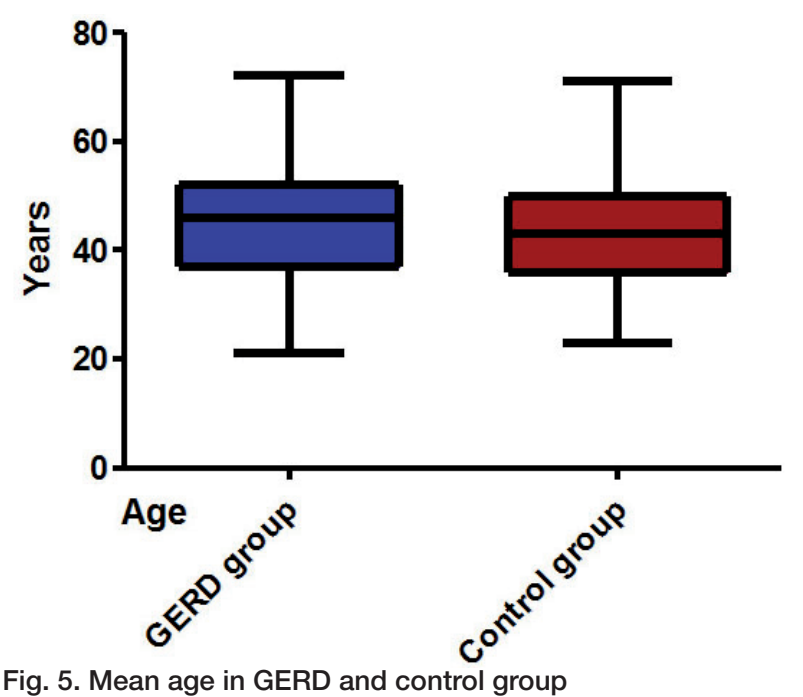

Fig. 5. Mean age in GERD and control group 
Analysis of the answers given by the respondents in the two groups are reflected in table I.

DMFT index in GERD group was $19.49 \pm 4.28$ (median=20) and in control group 18.16 $\pm 4.54($ median $=18)$, and value $\mathrm{p}=0.0581 \quad(\mathrm{p}<0.05)$ (Figure 6).

\section{Discussions}

Dental caries are a serious concern in our country, part because there are no well-established prevention programs and because of the small interest of a certain category of individuals in the population regarding oral health. The individuals suffering from a systemic disease are more concerned about general health than oral health, without taking into consideration the consequences of poor oral health.

It is a known fact that patients diagnosed with gastroesophageal reflux disease present specific oral manifestations. Some of these patients are not aware that the oral manifestations are consequences of gastroesophageal reflux disease and they do not have a proper oral hygiene or do not regularly visit a dentist.

The most frequent GERD dental complication is represented by an increased number of dental erosions (11), which is why most of the clinical studies researched the occurrence and the evolution of dental erosions in gastroesophageal reflux. There are few studies that followed the occurrence of dental caries in this medical disorder.

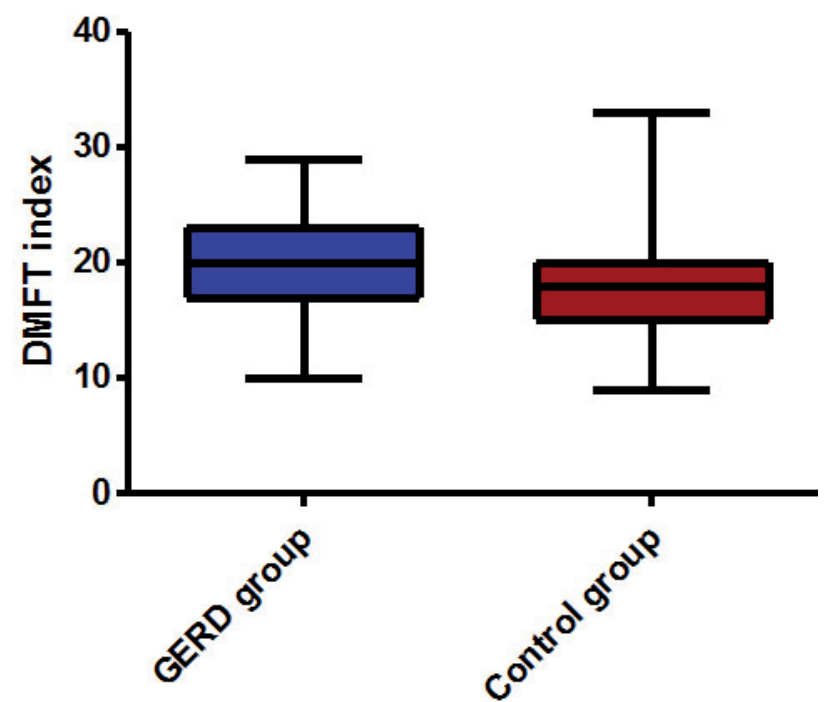

Fig. 6. DMFT index in GERD and control group

Therefore we considered that it is important to research if GERD patients are more likely to present more dental caries than the healthy individuals.

The individuals included in the two groups (GERD and control group), presented similar characteristics concerning age, gender and environment.

Regarding oral hygiene, most of the respondents from both groups stated that they brush their teeth once a

Table I. The frequency of answers on each question in GERD and control group

\begin{tabular}{|c|c|c|}
\hline & GERD group & Control group \\
\hline \multicolumn{3}{|c|}{ Please indicate how many times you brush your teeth } \\
\hline 1 time / day & $34(47.9 \%)$ & $39(61.9 \%)$ \\
\hline 2 times / day & $31(43.7 \%)$ & $19(30.2 \%)$ \\
\hline Few days a week & $3(4.2 \%)$ & $2(3.2 \%)$ \\
\hline I do not brush my teeth & $3(4.2 \%)$ & $3(4.8 \%)$ \\
\hline \multicolumn{3}{|l|}{ What type of toothbrush bristles do you use? } \\
\hline Extra soft & $2(2.9 \%)$ & $1(1.7 \%)$ \\
\hline Soft & $11(16.2 \%)$ & $13(21.7 \%)$ \\
\hline Medium & $30(44.1 \%)$ & $35(58.3 \%)$ \\
\hline Hard & $25(36.8 \%)$ & $11(18.3 \%)$ \\
\hline \multicolumn{3}{|l|}{ What oral care products do you use? } \\
\hline Toothpaste & $39(54.9 \%)$ & $37(58.7 \%)$ \\
\hline Toothpaste and mouthwash & $6(8.5 \%)$ & $10(15.9 \%)$ \\
\hline Toothpaste and dental floss & $15(21.1 \%)$ & $9(14.3 \%)$ \\
\hline Toothpaste, mouthwash and dental floss & $7(9.9 \%)$ & $4(6.3 \%)$ \\
\hline \multicolumn{3}{|c|}{ How often do you use other oral care products(mouthwash or dental floss)? } \\
\hline 1 time / day 1 & $20(28.2 \%)$ & $8(12.7 \%)$ \\
\hline 2 times / day & $2(2.8 \%)$ & $5(7.9 \%)$ \\
\hline Few days a week & $6(8.5 \%)$ & $10(15.9 \%)$ \\
\hline Never & $43(60.6 \%)$ & $40(63.5 \%)$ \\
\hline \multicolumn{3}{|l|}{ How often do you visit a dentist during a year? } \\
\hline 1 time / year & $13(18.3 \%)$ & $31(49.2 \%)$ \\
\hline 2 times / year & $14(19.7 \%)$ & $22(34.9 \%)$ \\
\hline Few times & $34(47.9 \%)$ & $5(7.9 \%)$ \\
\hline Only when I have a toothache & $10(14.1 \%)$ & $5(7.9 \%)$ \\
\hline \multicolumn{3}{|c|}{ Regarding dental status, what are your complains? } \\
\hline Sensitive teeth & $32(45.1 \%)$ & $20(31.7 \%)$ \\
\hline Caries & $13(18.3 \%)$ & $15(23.8 \%)$ \\
\hline Dental erosions & $41(57.7 \%)$ & $9(14.3 \%)$ \\
\hline
\end{tabular}


day, using a medium bristled toothbrush and only toothpaste, without using additional care products (mouthwash or dental floss). We noticed that the respondents in the GERD group are more aware that they need to visit a dentist, $47.9 \%$ of them stated that they are consulted by a dentist few times a year, while the respondents from the control group visit a dentist only once a year.

Regarding dental status, individuals in the GERD group complained more about sensitive teeth $(45.1 \%)$, dental erosions (57.7\%) and bleeding while brushing (71.8\%), compared with the control group. These data represents only the health status that the individuals were aware of.

The evaluation of dental caries was performed by a dentist blind to the diagnosis of the subjects, using DMFT index. After the consultation, there was no statistical differences ( $p>0.05$ ) noticed between DMFT index in the GERD and control group. The sensitive teeth and the bleeding while brushing could be explained by the use of medium or hard bristled toothbrush and no use of toothpaste for sensitive teeth. Similar results were obtained in a study conducted by Muňoz et al. (4), where it was compared CAO index in the control group (healthy volunteers) and GERD group $(p>0.05)$. Another study (3) showed no differences in the prevalence of dental caries in a group with gastroesophageal reflux patients and a control group.

Patients with GERD, even if they do not present a higher frequency of dental caries, still need to have a specific and proper oral hygiene, consisting in brushing twice a day, using extra soft or soft bristled toothbrush, toothpaste for sensitive teeth, a low abrasive fluoridated toothpaste, mouthwash and dental floss. Further on they need to regularly visit a dentist and they should not brush their teeth immediately after the exposure to acid; usually, approximately 60 minutes should pass until the saliva neutralizes the acids.

Patients diagnosed with GERD need an interdisciplinary treatment, both gastroenterological and dental.

As other researchers (12), we consider that untreated or continuing dental erosions will increase susceptibility to caries in the patients diagnosed with GERD. These patients, besides a proper oral hygiene, they also need an adequate dental treatment. In order to achieve a proper treatment it must be established the remineralization of erosion areas, which begins when the oral $\mathrm{pH}$ increases above 5.5. That is why the GERD patients must avoid carbonated and acidic beverages. Last but not least, these patients require fluoride treatments which offer a greater resistance to demineralization.

\section{Conclusions}

The subjects included in the study were similar regarding age, gender and environment to assure the comparability of the two groups.

Although the subjects in our study presented an increased number of dental erosions, there was no difference between GERD and control group regarding the frequency of dental caries, but untreated dental erosions might lead to dental caries or the loss of affected teeth.

Patients diagnosed with gastroesophageal reflux disease compared with the control group, complained more about sensitive teeth and bleeding while brushing, but the dental treatment in these patients must be an adequate one taking into consideration the diagnosis of GERD.

\section{Acknowledgment}

This paper is supported by the Sectoral Operational Programme Human Resources Development (SOP HRD), financed from the European Social Fund and by the Romanian Government under the contract number POSDRU/159/1.5/S/133377

\section{References}

1. V Linnett, WK Seow, F Connor, R Shepherd. Oral health of children with gastro-esophageal reflux disease: A controlled study. Australian Dental Journal 2002;47:156-162.

2. van Roekel NB. Gastroesophageal Reflux Disease, Tooth Erosion, and Prosthodontic Rehabilitation: A Clinical Report. J Prosthodont 2003;12:255-259.

3. Paraschiv C, Gavrilescu C, Cotea I, Paloma M, Ghiuru R. Extraesophageal manifestations of Gastroesophageal reflux disease with implications For oral cavity. Romanian Journal of Oral Rehabilitation, 2013;5:2.

4. Muñoz JV, Herreros B, Sanchiz V, Amoros C, Hernandez V, Pascual I, Mora F, Minguez M, Bagan JV, Benages A. Dental and periodontal lesions in patients with gastro-oesophageal reflux disease. Digestive and Liver Disease, 2003;35:461-467.

5. Correa MC, Lerco MM, da Cuhna ML, Henry MA. Salivary parameters and teeth erosions in patients with gastroesophageal reflux disease. Arq Gastroenterol, 2012;49:214-218.

6. Davies A, Sandhy B. Diagnosis and treatment of gastroesophageal reflux. Arch Dis Child 1995;73:82-86.

7. Fonkalsrud E, Ament M. Gastresophageal reflux in childhood. Curr Probl Surg 1996;33:31-70.

8. Romano and Cardile: Gastroesophageal reflux disease and oral manifestations. Italian Journal of Pediatrics, 2014;40(Suppl 1):A73.

9. Moshkowitz M, Horowitz N, Halpern Z, Santo E. Gastroesophageal reflux disease symptoms: Prevalence, sociodemographics and treatment patterns in the adult Israeli population. World J Gastroenterol, 2011;17:1332-1335.

10. Chi AC, Neville BW, Krayer JW, Gonsalves WC. Oral manifestations of systemic disease. Am Fam Physician, 2010,82:1381-1388.

11. Alavi G, Alavi AA, Saberfiroozi M, Sarbazi AH, Motamedi M. Dental erosion in patients with gastro esophageal refluxdisease (GERD). Journal of Dentistry and Oral Hygiene, 2013;5:62-67.

12. Barron RP, Carmichael RP, Margaret Marcon, Sandor GKB. Dental erosion in gastroesophageal reflux disease. J Can Dent Assoc 2003;69:84-89. 